\title{
The Cdc2/Cdk1 inhibitor, purvalanol A, enhances the cytotoxic effects of taxol through Op18/stathmin in non-small cell lung cancer cells in vitro
}

\author{
XIAN CHEN ${ }^{1}$, YING LIAO ${ }^{1}$, DAN LONG ${ }^{1}$, TING YU ${ }^{1}$, FANG SHEN ${ }^{2}$ and XUECHI LIN ${ }^{1,3}$ \\ ${ }^{1}$ Department of Medical Laboratory, Changsha Medical University, Changsha, Hunan 410219; ${ }^{2}$ Department of Clinical Laboratory, \\ The First Affiliated Hospital of Hunan Normal University, Changsha, Hunan 410005; ${ }^{3}$ Department of Anatomy, Histology \\ and Embryology, Institute of Neuroscience, Changsha Medical University, Changsha, Hunan 410219, P.R. China
}

Received August 1,2016; Accepted May 3, 2017

DOI: $10.3892 /$ ijmm.2017.2989

\begin{abstract}
Purvalanol A is a highly selective inhibitor of Cdc2 [also known as cyclin-dependent kinase 1 (CDK1)]. Taxol is an anti-tumor chemotherapeutic drug which is widely used clinically. In this study, the CDK1 inhibitor, purvalanol A was applied to explore the relevance of $\mathrm{Cdc} 2$ signaling and taxol sensitivity through analyses, such as cellular proliferation and apoptosis assays, ELISA, western blot analysis and immunoprecipitation. We demonstrated that purvalanol A effectively enhanced the taxol-induced apoptosis of NCI-H1299 cells, as well as its inhibitory effects on cellular proliferation and colony formation. In combination, purvalanol A and taxol mainly decreased the expression of oncoprotein 18 (Op18)/ stathmin and phosphorylation at Ser16 and Ser38, while purvalanol A alone inhibited the phosphorylation of Op18/stathmin at all 4 serine sites. Co-treatment with purvalanol $\mathrm{A}$ and taxol weakened the expression of Bcl-2 and activated the extrinsic cell death pathway through the activation of caspase- 3 and caspase- 8 . Further experiments indicated that $\mathrm{Cdc} 2$ kinase activities, including the expression of $\mathrm{Cdc} 2$ and the level of phospho-Cdc2 (Thr161) were significantly higher in taxolresistant NCI-H1299 cells compared with the relatively sensitive CNE1 cells before and following treatment with taxol. These findings suggest that $\mathrm{Cdc} 2$ is positively associatd with the development of taxol resistance. The $\mathrm{Cdc} 2$ inhibitor, purvalanol A, enhanced the cytotoxic effects of taxol through Op18/stathmin. Our findings may prove to be useful in clinical practice, as they may provide a treatment strategy with which to to reduce the doses of taxol applied clinically, thus alleviating the side-effects.
\end{abstract}

Correspondence to: Professor Xuechi Lin, Department of Medical Laboratory, Changsha Medical University, Leifeng Road, Wangcheng, Changsha, Hunan 410219, P.R. China

E-mail: xuechilin71@126.com

Key words: Cdc2, purvalanol A, taxol, oncoprotein 18/stathmin, non-small cell lung cancer

\section{Introduction}

Cdc2, also known as cyclin dependent kinase 1 (CDK1), controls the cell cycle entry from the G2 to the M phase and promotes the commencement of mitotic phase events (1), the abnormal activation of which directly causes aberrant cell proliferation, and malignant transformation and tumorigenesis in prostate cancer cells (2-4). Cdc2 activation also depends on the phosphorylation of Thr161, and CDC25-mediated dephosphorylation at Thr14 and Tyr-15, which exhibits enzymatic activity when only phospho-Thr161 remains (5).

Purvalanol $\mathrm{A}$ is a selective inhibitor of $\mathrm{Cdc} 2$, which strongly inhibits $\mathrm{Cdc} 2$ kinase activity at a low concentration of $2 \mu \mathrm{M}(6,7)$. Investigators have identified that purvalanol $\mathrm{A}$ effectively suppresses Cdc2 activity and the progression from the $\mathrm{G} 2$ phase to mitosis, which leads to the loss of clonogenicity and cellular apoptosis in both MKN45 and MKN28 $\mathrm{X}$-irradiated gastric cancer cells (8).

Oncoprotein 18 (Op18)/stathmin is a small molecule weight phosphoprotein which is highly expressed in cancer cells. Its main functions are to regulate the equilibrium of microtubule (MT) dynamics and control cell cycle progression, which is closely associated with the maintenance of tumor malignant phenotypes (9-12). Op18/stathmin has 4 phosphoserine sites (p-Ser16, p-Ser25, p-Ser38 and p-Ser63), which integrates and relays various signals from intra- or extacellular stimuli through phosphorylated inactivation and dephosphorylated activation (13-15).

In a previous study, we found that Epstein-Barr virusspecific protein-latent membrane protein 1 (LMP1) regulates the Op18/stathmin signaling pathway by mediating $\mathrm{Cdc} 2$, which accelerates cell cycle progression and promotes cell proliferation (16). In another recent study of ours, we confirmed that human NCI-H1299 non-small cell lung cancer cells highly expressing Op18/stathmin were the most highly resistant to taxol among 5 different cancer cells originating from epithelia, including CNE1, Hep3B-2, MGC, MCF-7 and NCI-H1299 (17).

In this study, NCI-H1299 cells were employed to clarify the association between $\mathrm{Cdc} 2$ signaling and taxol resistance, and to elucidate the related molecular mechanisms. 


\section{Materials and methods}

Cells and cell growth conditions. Both the CNE1 cells and NCI-H1299 cells were a kind gift from Professor Ya Cao from the Cancer Research Institute of Central South University and cultured in RPMI-1640 (Gibco-BRL, Grand Island, NY, USA) with $10 \%$ fetal bovine serum (FBS; HyClone, Logan, UT, USA), $100 \mathrm{IU} / \mathrm{ml}$ penicillin and $100 \mu \mathrm{g} / \mathrm{ml}$ streptomycin at $37^{\circ} \mathrm{C}, 5 \% \mathrm{CO}_{2}$.

CNE1 human cancer cells were testified to be the most sensitive to taxol among 5 various epithelial-deriving tumor cells in our previous study (17). Thus, they were used in this study for a comparison to the NCI-H1299 cells.

Antibodies andreagents. The primary antibodies used were antistathmin (Cat. no. 569391; Calbiochem, Billercia, CA, USA), anti-phosphoserine (Cat. no. 618100; Zymed, San Francisco, CA, USA), anti-Cdc2 (sc-954; Santa Cruz Biotechnology, Inc., Santa Cruz, CA, USA), anti-phospho-Thr161-Cdc2 (Cat. no. 9114; Cell Signaling Technology, Danvers, MA, USA), anti-cyclin B1 (sc-7393), anti-caspase-3 (sc-7272) (both from Santa Cruz Biotechnology, Inc.), anti-caspase-8 (Cat. no. 9746), rabbit monoclonal anti-caspase-9 (Cat. no. 9502) (both from Cell Signaling Technology), anti-phospho-stathmin [phospho-S16 (ab47328), S25 (ab194752), S38 (ab194757), S63 (ab76583); Abcam, Cambridge, MA, USA], anti-Bcl-2 (sc-492; Santa Cruz Biotechnology, Inc.), anti-extracellular signalregulated kinase 1 (ERK1; sc-93), anti-phospho-ERK1 (sc-7383) (both from Santa Cruz Biotechnology, Inc.), anti- $\beta$-actin (Cat. no. A2228; Sigma, St. Louis, MO, USA). The secondary antibodies used where horseradish peroxidase (HRP)-conjugated goat anti-rabbit IgG (sc-2004) and rabbit anti-mouse IgG (sc-358914) (both from Santa Cruz Biotechnology, Inc.).

Taxol (Santa Cruz Biotechnology, Inc.) and purvalanol A (Calbiochem) were both purchased and dissolved in dimethyl sulfoxide (DMSO) as mother solutions at $-20^{\circ} \mathrm{C}$ for use.

Flow cytometric analysis. The cells were plated in 6-well plates, and pre-treated with 0,1 and $5 \mu \mathrm{M}$ purvalanol A for $2 \mathrm{~h}$ when they reached $80 \%$ confluency. This was followed by the addition of $100 \mathrm{nM}$ taxol for $12 \mathrm{~h}$. DMSO was used as a solvent control. The cells were then rinsed with phosphate-buffered saline (PBS) and digested with $0.25 \%$ trypsin.

The cells were cropped by centrifugation and stained with a mixture of $5 \mu \mathrm{l}$ propidium iodide (PI) and $10 \mu \mathrm{l}$ Annexin V-FITC according to the protocol provided with the Annexin V-FITC Apoptosis Detection kit (Nanjing Biobox Biotech. Co, Ltd, Nanjing, China).

Cellular apoptosis was assessed by flow cytometry (FCM) by a specialized agency (the Second Xiangya Hospital Affiliated Central South University). All experiments were performed in triplicate.

MTT assay. The cells at the logarithmic phase were seeded at 5,000 cells/well in 96-well plates and pre-treated with various concentrations of purvalanol $\mathrm{A}(0,1$ and $5 \mu \mathrm{M})$ for $2 \mathrm{~h}$, followed by the addition of $100 \mathrm{nM}$ taxol. Finally, $10 \mu \mathrm{l}$ of 3-(4,5-dimethylthiazol-2-yl)-2,5-diphenyl (MTT) were added at the specified time points of 24,48 and $72 \mathrm{~h}$. The values of optical density (OD) at a wavelength $570 \mathrm{~nm}$ were then detected using a microplate reader (BioTek, Winooski, VT, USA). Cell proliferatoin was calculated using the following formula: relative proliferation rates $(\%)=(\mathrm{OD}$ treatment $/ \mathrm{OD}$ control) $x 100 \%$. Six parallel wells were set in each group.

Colony formation assay. A total of 2,500 cells in a single suspension were plated per well in a 6-well plate and divided into 6 groups, including 3 co-treatment groups and 3 gradient groups of purvalanol A alone. Cell growth was terminated when colonies were observed by the naked eye after 2 weeks. Colonies containing over 50 cells were counted using an inverted microscope, and images were acquired using a DMCI microscope (Leica, Wetzlar, German) at x200 magnification. All experiments were performed in triplicate.

Assessment of Cdc2 kinase activity. Assays to determine Cdc2 kinase activity were performed according to the instructions provided with the MESACUP Cdc2 kinase assay kit (code no. 5235; MBL, Nagoya, Japan). Cells reaching $80 \%$ confluence were treated with various concentrations of taxol $(0,10$ and $100 \mathrm{nM})$ for $12 \mathrm{~h}$, then lysed in a sample buffer. The supernatants were then collected for the detection of Cdc2 kinase activity as described in our previous study (16). ELISA was performed according to the instructions provided with the MESACUP Cdc2 Kinase Assay kit. Briefly, $100 \mu \mathrm{l}$ of cell extracts were transferred to a microwell strip coated with monoclonal antibody 4A4 (4 parallel wells/sample), incubated at $25^{\circ} \mathrm{C}$ for $60 \mathrm{~min}$ and washed 5 times. This was followed by the addition of $100 \mu \mathrm{l}$ peroxidase (POD) conjugated streptavidin, and incubation at $25^{\circ} \mathrm{C}$ for $30 \mathrm{~min}$ and washing 5 times. The cells were then treated with $100 \mu \mathrm{l}$ POD substrate solution for $5 \mathrm{~min}$, and the reaction was then terminated with $100 \mu \mathrm{l}$ Stop Solution (20\% phosphoric acid). The OD value was read at a $490 \mathrm{~nm}$ wavelength using a microplate reader (BioTek). All experiments were carried out in triplicate.

Western blot analysis. Following the removal of the supernatant, the cells were lysed in cell lysis buffer $(50 \mathrm{mM}$ Tris- $\mathrm{HCl} \mathrm{pH} \mathrm{8.0,}$ $1 \mathrm{mM}$ EDTA, 2\% SDS, $5 \mathrm{mM}$ DTT and $10 \mathrm{mM}$ PMSF). Total proteins $(50 \mu \mathrm{g})$ were then separated by $10 \%$ sodium dodecyl sulfate-polyacrylamide gel electrophoresis (SDS-PAGE) and transferred onto nitrocellulose membranes and incubated with the specific primary antibodies at $4^{\circ} \mathrm{C}$ overnight, followed by the addition of HRP conjugated secondary antibodies for $2 \mathrm{~h}$ at room temperature. An enhanced chemiluminescence detection kit (Pierce, Rockford, IL, USA) was applied for immunoblotting.

Immunoprecipitation (IP) - western blot analysis. In brief, cell extracts were collected in IP lysis buffer, as described in our previous studies $(16,18)$. Immunoprecipitates of Op18/ stathmin were pulled down by excessive anti-stathmin antibody binding magnetic immunobeads and separated by 10\% SDS-PAGE. anti-phosphorserine antibody was used to detect the total level of phosphorylated Op18/stathmin by western blot analysis.

Statistical analysis. Statistical analysis was carried out using SPSS 17.0 statistical software. The specific statistical method 


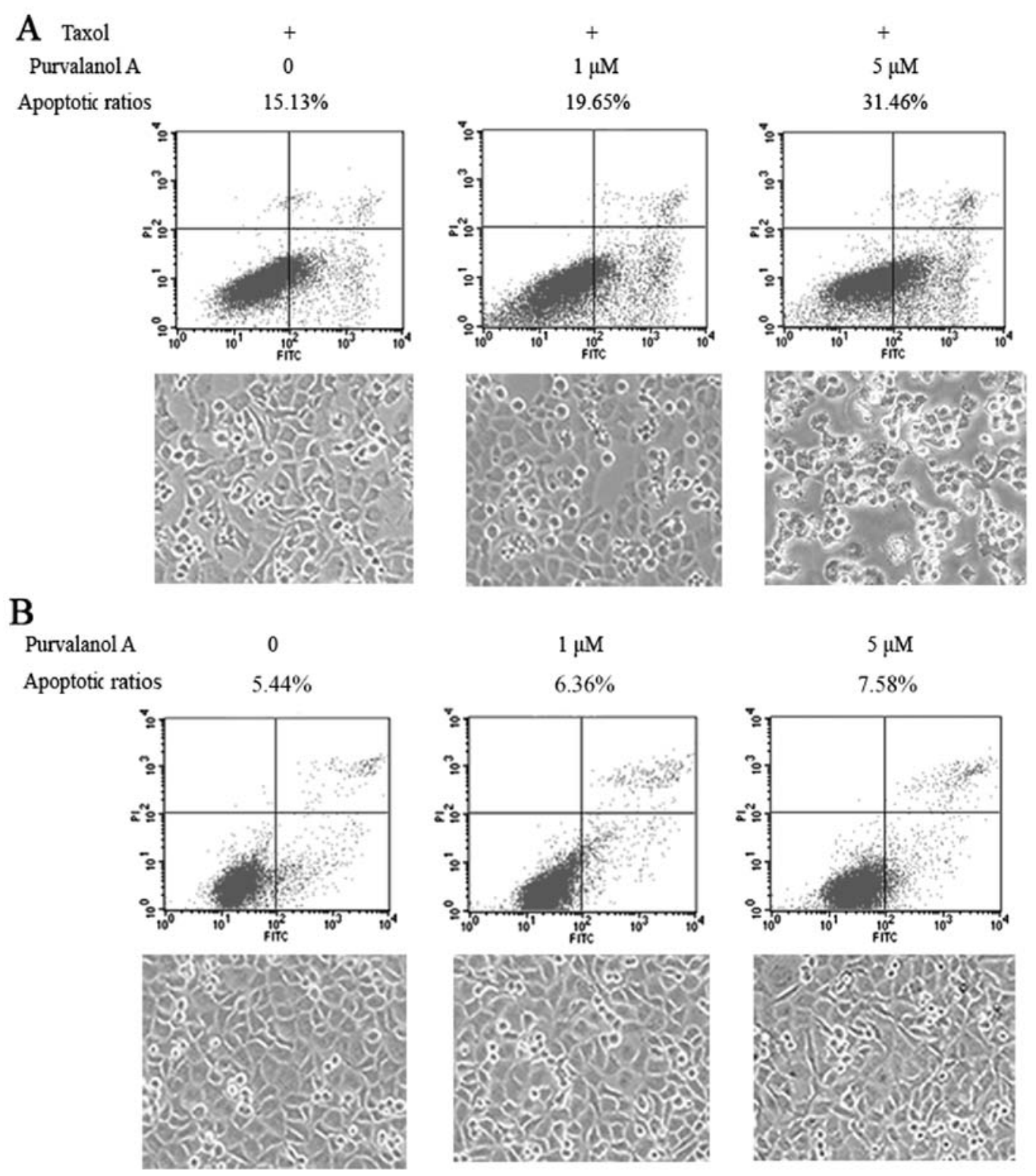

Figure 1. Purvalanol A promotes taxol-induced cellular apoptosis and growth inhibition. (A) Upper panel shows cellular apoptotic ratios, and the images in the lower panel represent the results of cellular growth. (B) Status of cellular apoptosis and growth in cells treated with purvalanol A alone at increasing concentrations.

applied was the t-test, and a value of $p<0.05$ was considered to indicate a statistically significant difference. All data are presented as the means $\pm \mathrm{SD}$.

\section{Results}

Purvalanol A enhances the taxol-induced apoptosis of NCI-H1299 cells. The cellular apoptotic ratios were 15.13, 19.65 and $31.46 \%$ in the NCI-H1299 cells treated with taxol in presence of 0,1 and $5 \mu \mathrm{M}$ purvalanol A, respectively. Purvalanol A augmented taxol-induced cellular apoptosis in a concentration dependent manner. The images of cellular growth revealed that a greater number of translucent floating cells appeared in the medium of the cells treated with purvalanol A (Fig. 1A).

In the cells treated with purvalanol $\mathrm{A}$ alone at concentrations of 0,1 and $5 \mu \mathrm{M}$, the cellular apoptotic ratios were 5.44, 6.36 and $7.58 \%$, respectively. These cells almost reached complete confluence and there were only a few translucent floating cells (Fig. 1B).

Purvalanol A enhances the inhibitory effects of taxol on cellular proliferation and colony formation in NCI-H1299 cells. Purvalanol A enhanced the inhibitory effects of taxol on cellular proliferation in NCI-H1299 cells. In the cells co-treated with $5 \mu \mathrm{M}$ purvalanol A and taxol, the representative curve steeply descended at the time points of 24, 48 and $72 \mathrm{~h}$; the relative proliferation ratios were $80.53,59.01$ and $41.12 \%$, respectively. In the cells co-treated with $1 \mu \mathrm{M}$ purvalanol A and taxol, the representative curve descended slowly, with proliferation ratios of $94.98,89.6$ and $78.32 \%$ at 24,48 and $72 \mathrm{~h}$, respectively (Fig. 2A).

The histograms indicated that a statistically significant difference existed between the cells co-treated with $5 \mu \mathrm{M}$ purvalanol A and taxol, and the other 2 groups (taxol only, 
A

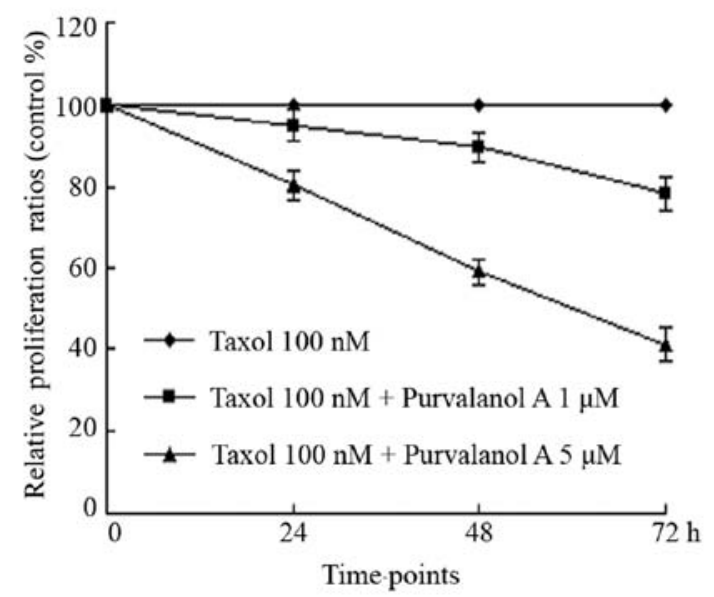

C

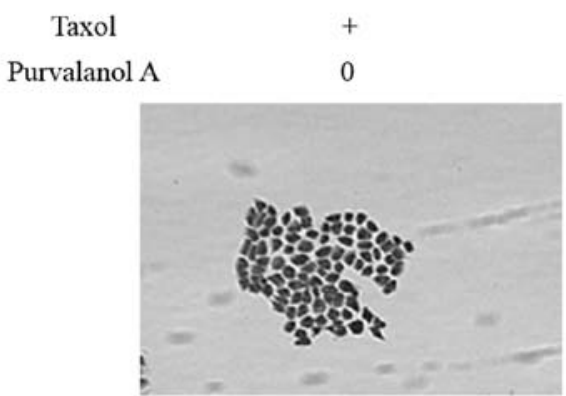

Purvalanol A

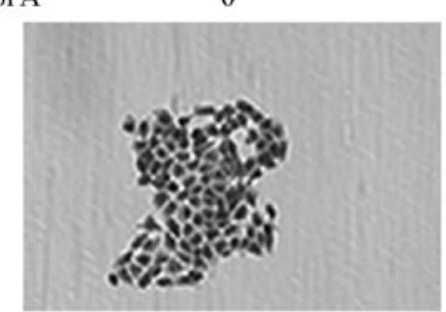

B a Taxol $100 \mathrm{nM}$

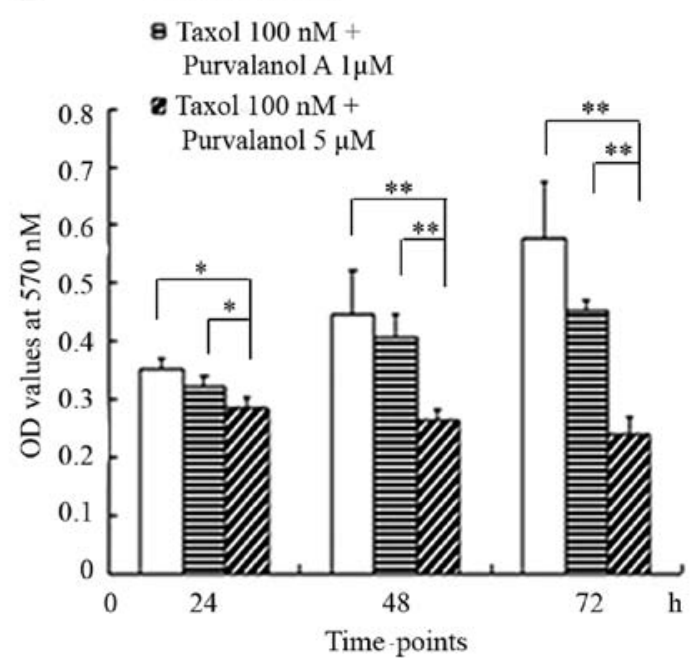

$+$

$1 \mu \mathrm{M}$

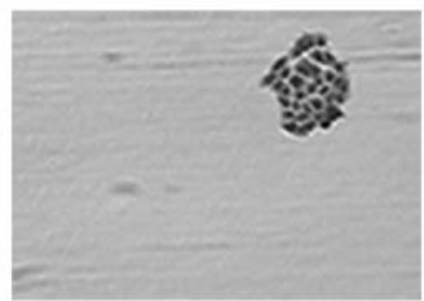

$1 \mu \mathrm{M}$

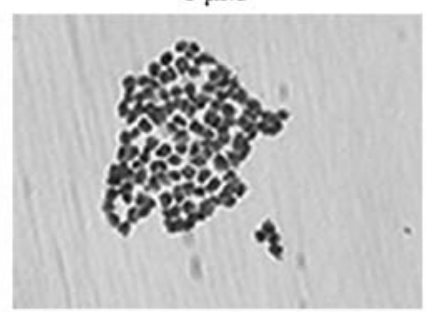

Time-points

\section{.}
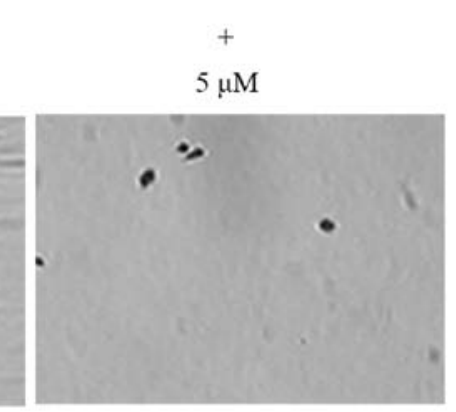

$5 \mu \mathrm{M}$

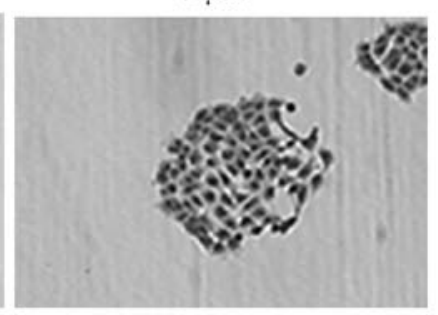

Figure 2. Combination of purvalanol A and taxol suppresses cellular proliferation and colony formation. (A) Curves represent proliferation trends at 3 time points. (B) Histograms show statistically significant differences. ${ }^{*} p<0.05$ and ${ }^{* *} p<0.01$. (C) Images show the capacities of colony formation. Original magnification, $x 200$.

and taxol $+1 \mu \mathrm{M}$ purvalanol $\mathrm{A})$ at $24 \mathrm{~h}(\mathrm{p}<0.05)$, while the differences became more and more significant with the passing of time (p<0.01) (Fig. 2B).

Only a few and sparse small colonies appeared in the group treated with $1 \mu \mathrm{M}$ purvalanol A combined with taxol; no visibly typical colonies were formed in the group co-treated with $5 \mu \mathrm{M}$ purvalanol $\mathrm{A}$ and taxol, while a large number of colonies appeared among the group treated with taxol only and in the 3 control groups treated with purvalanol A only at increasing concentrations, which usually merged into a large colony with ambiguous borders (Fig. 2C).

Co-treatment with purvalanol $A$ and taxol inhibits the expression of Opl8/stathmin and phoshorylation. The expression of Op18/stathmin was weakened in the NCI-H1299 cells treated with a combination of purvalanol A and taxol; the synergistic inhibitory effects were the most evident in the cells treated with a combination of $5 \mu \mathrm{M}$ purvalanol $\mathrm{A}$ and taxol in comparison with the other 2 groups (Fig. 3A). Treatment with taxol alone slightly decreased the expression of Op18/stathmin at the concentration of $100 \mathrm{nM}$; however, no notable alternation in the expression of Op18/stathmin was observed in the cells treated with purvalanol $\mathrm{A}$ at increasing concenrations (Fig. 3B).

IP analysis revealed that the total levels of phosphoOp18/stathmin were notably decreased in the cells co-treated with purvalanol A and taxol, compared with the controls or the cells treated with taxol or purvalanol A alone (Fig. 3C).

Treatment with purvalanol A alone inhibited the phosphorylation of Op18/stathmin at all 4 serine sites, including Ser16, Ser25, Ser38 and Ser63 compared with the cells treated with taxol alone. Treatment with both purvalanol A and taxol mainly inhibited the phosphorylation of Op18/stathmin at Ser16 and Ser38 compared with the cells treated with purvalanol A alone; however, the phosphorylation levels of Ser25 and Ser63 sites were similar between the 2 groups (Fig. 3D). 


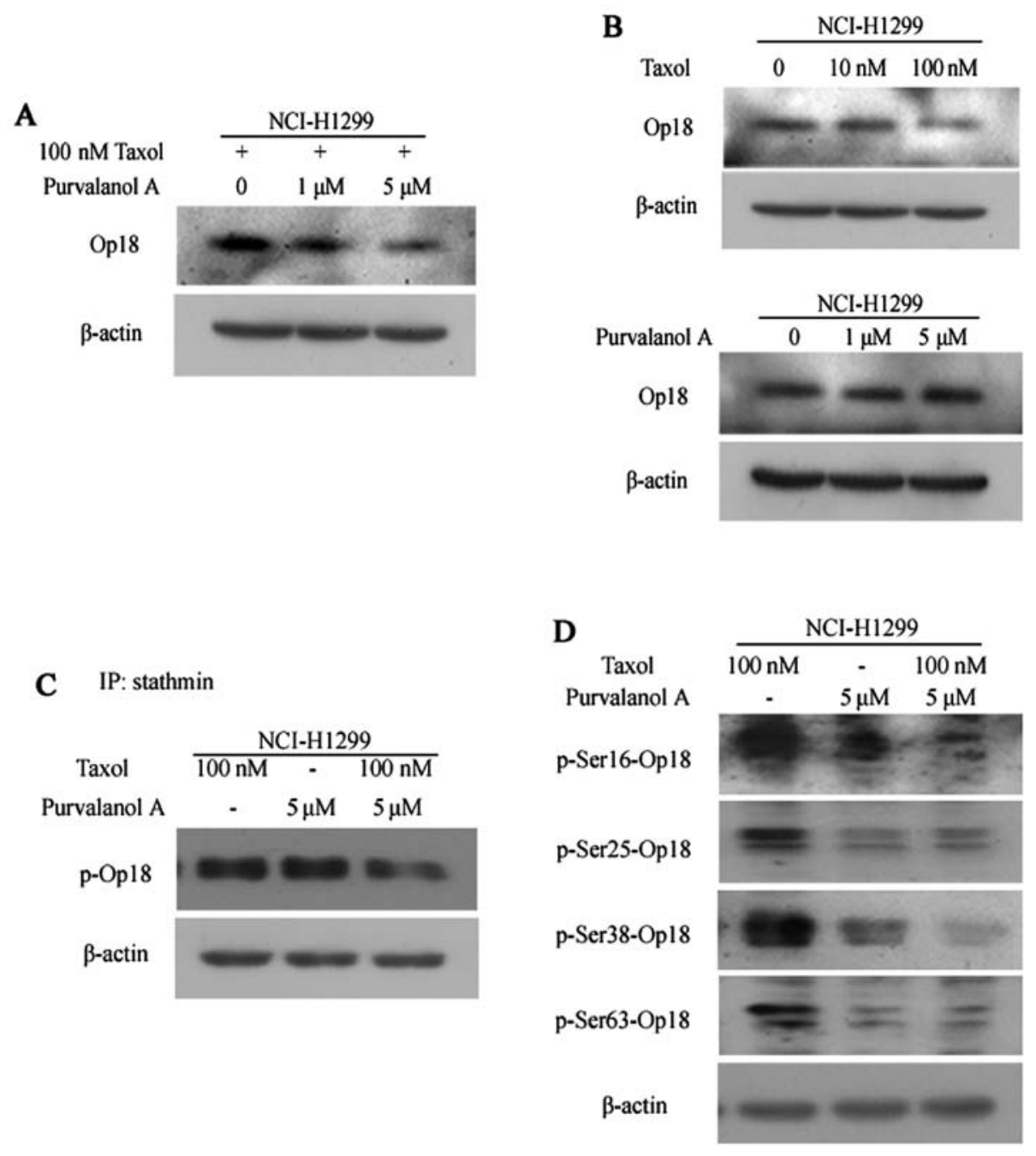

Figure 3. Purvalanol A combined with taxol jointly inhibits the expression of oncoprotein 18 (Op18)/stathmin and phoshorylation. (A and B) The expression of Op18/stathmin was detected by western blot analysis. (C) Immunoprecipitation (IP) - western blot was used to detect the total levels of phospho-Op18/stathmin.

(D) Purvalanol A combined with taxol mainly inhibited the phosphorylation of Op18/stathmin at the Ser16 and Ser38 sites.

Treatment with both purvalanol $A$ and taxol decreases the expression of Bcl-2 and initiates the activation of caspase-3 and caspase-8. The expression and phosphorylation of ERK was not altered in the cells treated with both purvalanol A and taxol. However, combination treatment markedly inhibited the expression of $\mathrm{Bcl}-2$, with a greater decrease observed with increasing concentrations of purvalanol A (Fig. 4A). No differences were observed in the expression of these molecules in the cells treated with purvalanol A alone at increasing concentrations (Fig. 4B).

Purvalanol A and taxol collectively increased the expression levels of caspase-3 and caspase- 8 with a greater increase observed with increasing concentrations of purvalanol A. No obvious changes were observed in the levels of caspase-9 (Fig. 4C). Similarly, treatment with purvalanol A alone at increasing concentrations did not lead to any alternations in the levels of caspase- 3 , caspase- 8 and caspase- 9 in the NCI-H1299 cells (Fig. 4D).

Cdc2 is positively associated with the development of taxol resistance in different epithelia-derived tumor cells. The histograms indicated that Cdc2 kinase activity was markedly higher in the NCI-H1299 cells compared with the CNE1 cells before and after treatment with taxol. Taxol also downregulated the activity of $\mathrm{Cdc} 2$ to a certain extent in a concentrationdependant manner in both cell lines (NCI-H1299 and CNE1), although this decrease in the levels $\mathrm{Cdc} 2$ was more evident in the CNE1 cells in comparison to the NCI-H1299 cells; the differences became more significant with the addition of Taxol (p<0.01) (Fig. 5A).

The results of western blot analysis revealed that the expression levels of $\mathrm{Cdc} 2$ and phosporylation at the Thr161 site were markedly higher in the NCI-H1299 cells than in the CNE1 cells before and after treatment with taxol. Treatment with taxol alone did not affect the expression of $\mathrm{Cdc} 2$, but slightly decreased the phosphorylation of Cdc2-Thr161 in both cell lines, which was coincident with the analysis of Cdc2 kinase activity by enzyme-linked immunosorbent assay (ELISA) (Fig. 5B).

FCM analysis revealed that the cellular apoptotic ratios were 12.53 and $34.94 \%$ in the NCI-H1299 cells and CNE1 cells treated with taxol, while the cellular apoptotic ratios were 5.02 and $5.49 \%$ in the controls treated with solvent DMSO; the status of cell growth shown in the images correlated with the findings of apoptotic analysis (Fig. 5C and D). 

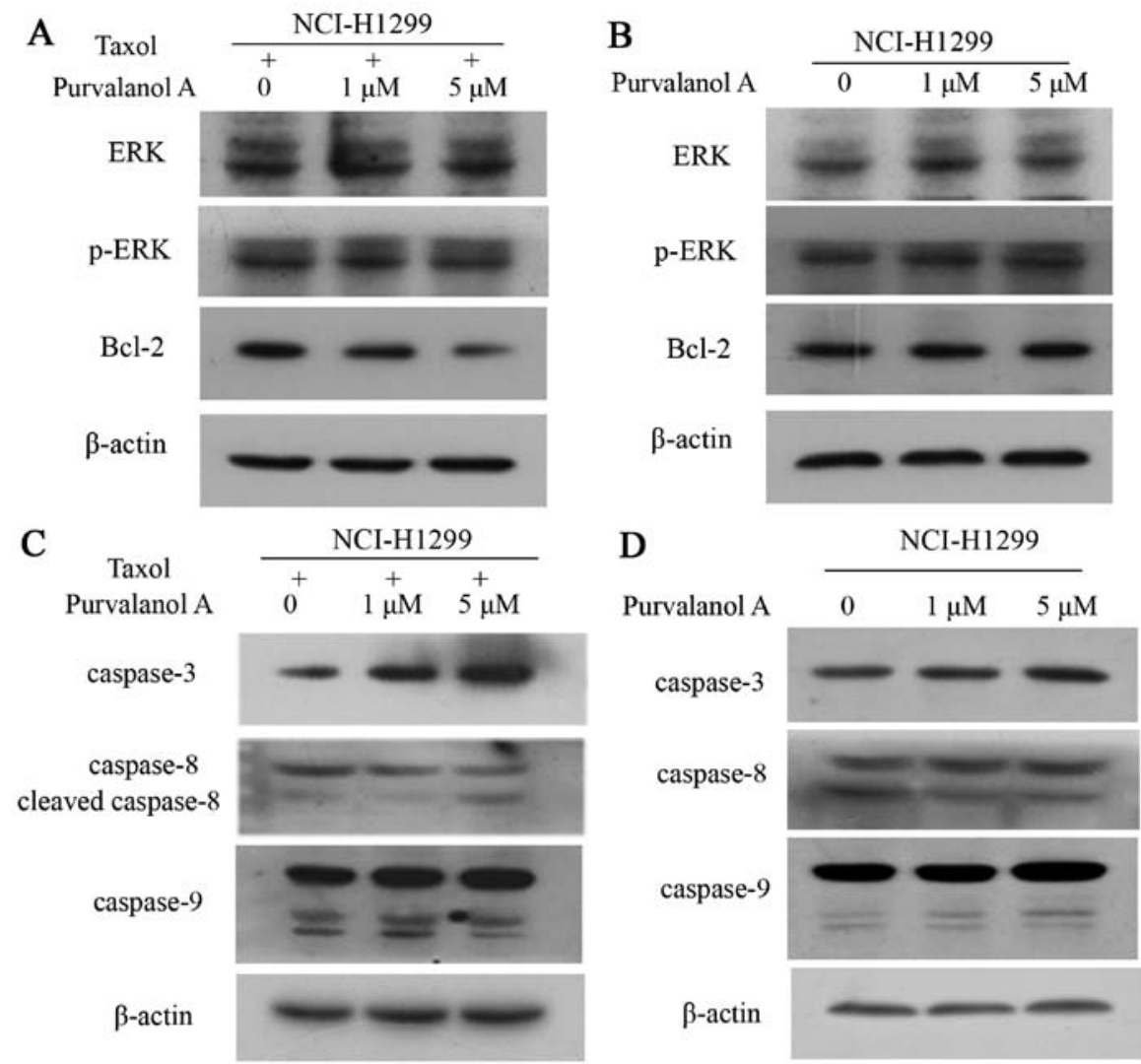

Figure 4. Combination of purvalanol A and taxol decreases the expression of $\mathrm{Bcl}-2$ and initiates the activation of caspase- 3 and caspase- 8 , while the expression of extracellular signal-regulated kinase (ERK) and p-ERK was not affected. (A) Cells were treated with a combination of purvalanol A and taxol. (B) Cells treated with purvalanol A at increasing concentrations were used as controls. (C) Status of caspase-3, caspase-8 and caspase-9 was detected in the co-treated groups . (D) No any obvious changes were observed in the cells treated with purvalanol A alone at increasing concentrations.
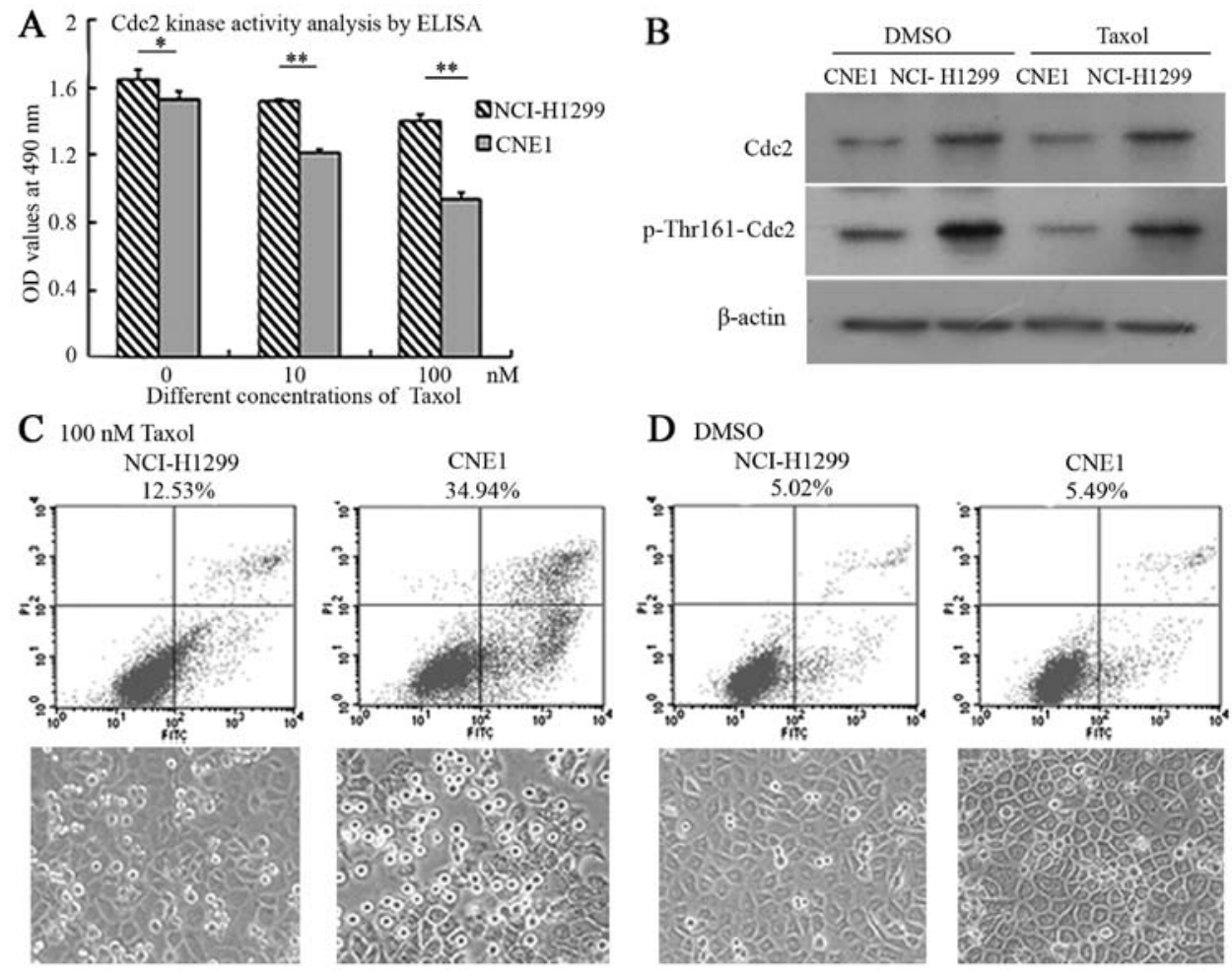

Figure 5. Cdc2 is positively associated with the development of taxol resistance in different epithelia-derived tumor cells. (A) Histograms show the differences in Cdc2 kinase activity by enzyme-linked immunosorbent assay (ELISA) between the NCI-H1299 cells and CNE1 cells. " $\mathrm{p}<0.05$ and ${ }^{* *} \mathrm{p}<0.01$. (B) Western blot analysis was used to detect the expression of Cdc2 and the level of p-Thr161-Cdc2. (C) Upper panel shows cellular apoptotic ratios in cells treated with taxol, and the images in the lower panel illustrate the status of cell growth. (D) Upper panel shows apoptotic ratios in different tumor cells treated with solvent dimethyl sulfoxide (DMSO), and the images in the lower panel indicate the growth situation of control cells. 


\section{Discussion}

Taxol is an effective antitumor chemotherapeutic agent derived from plants, which induces cell cycle arrest and cellular apoptosis by promoting MT polymerization (19-21). With the wide application of taxol in clinical practice, a large number of resistant tumors have emerged; therefore, high doses of taxol are increasingly prescribed, which results in severe side-effects. To date, no antagonizing agents have been identified (22). To minimize the dosage of taxol, the combined use of taxol with other traditional drugs has been used in an attempt to improve taxol sensitivity $(23,24)$. These strategies have achieved some success, but also lead to new risks of acquiring multidrug resistance.

In this study, we demonstrated that the Cdc2/CDK 1 inhibitor, purvalanol A, effectively enhanced the sensitivity of NCI-H1299 cells to taxol and inhibited cellular proliferation and colony formation. Other studies have also confirmed that CDK1 inhibition significantly enhances drug-induced colony formation and apoptosis in breast cancer cells and colon carcinoma cells and glioma cells (25-27).

Op18/stathmin is a downstream molecular target of Cdc2, and tumor cells which highly express Op18/Stathmin are resistant to taxol $(17,28)$. This study certified that the combination of purvalanol A and taxol mainly inhibited the expression of Op18/stathmin and phosphorylation at the Ser16 and Ser38 sites, while purvalanol A alone uniformly inhibited the phosphorylation of Op18/stathmin at all 4 serine sites. Other studies have shown that Cdc2 predominantly phosphorylates Op18/stathmin at Ser25 and Ser38 sites, which is partly different from our results, which may implicate other inhibitory activities of purvalanol A $(6,29)$.

$\mathrm{Bcl}-2$ is an anti-apoptotic factor involved in the resistance of conventional drugs. The overexpression of Bcl-2 potentially induces the development of taxol resistance in lung cancer cells (30). ERK is also an upstream kinase of Op18/stathmin, and ERK-mediated Op18/stathmin signaling also complicates to cell cycle progression and taxol sensitivity $(18,31)$. Cellular apoptosis is closely associated with the activation of caspase- 3 , caspase- 8 and caspase-9. As previously demonstrated, the blocking of ERK and taxol jointly induced exogenous cellular apoptosis via the upregulation of the expression of caspase- 3 and caspase- 9 in NCI-H1299 cells; the activation of caspase-3 and caspase-8 promoted cellular apoptosis and autophagy in A549 human lung cancer cells exposed to Paris polyphylla steroidal saponins (PPSS); paclitaxel and Op18 silencing collectively induced cell death through initiating caspase- 3 and caspase-9 activations in nasopharyngeal carcinoma CNE1 cells $(18,32,33)$. This study indicated that co-treatment with purvalanol A and taxol decreased the expression of Bcl-2 and initiated the activation of the extrinsic cell death pathway through the activation of caspase- 3 and caspase- 8 , but did not markedly affect the expression of ERK and phosphorylation.

We further found that both the expression of $\mathrm{Cdc} 2$ and the level of p-Thr161-Cdc2 was markedly higher in the NCI-H1299 cells in contrast with the CNE1 cells, which was consistent with the detection of Cdc2 kinase activity, while the NCI-H1299 cells with a high $\mathrm{Cdc} 2$ kinase activity exhibited obvious resistance to taxol in comparison with the relatively sensitive CNE1 cells, which implied that $\mathrm{Cdc} 2$ kinase activity is positively associated with the development of taxol resistance in different epithelia- derived cancer cells. Another study also demonstrated that a high expression of Cdc2 was negatively associated with the curative effects of chemotherapeutics and was a poor prognostic factor in epithelial-derived ovarian cancer and laryngeal squamous cell carcinoma $(34,35)$.

In conclusion, the findings of our study suggest that purvalanol A enhances the cytotoxic effects of taxol through Op18/stathmin in non-small cell lung cancer. Our findings may prove to be helpful in reducing the doses of taxol applied clinically and to alleviate the side-effects.

\section{Acknowledgements}

The present study was supported by grants from the National Natural Science Foundation of China (no. 81272274) and the Key Project of Hunan Province Scientific Research of Colleges and Universities (no. 12A018). The authors would like to thank Dr Liu Sufang from the Second Xiangya Hospital Affiliated Central South University for cellular apoptosis detection and Dr Tao Yongguang from the Cancer Research Institute of Central South University for providing valuable suggestions for the manuscript.

\section{References}

1. Zhao XF and Gartenhaus RB: Phospho-p70S6K and cdc2/cdk1 as therapeutic targets for diffuse large B-cell lymphoma. Expert Opin Ther Targets 13: 1085-1093, 2009.

2. Liu P, Kao TP and Huang H: CDK1 promotes cell proliferation and survival via phosphorylation and inhibition of FOXO1 transcription factor. Oncogene 27: 4733-4744, 2008.

3. Malumbres $\mathrm{M}$ and Barbacid M: Cell cycle, CDKs and cancer: A changing paradigm. Nat Rev Cancer 9: 153-166, 2009.

4. Pérez de Castro I, de Cárcer G and Malumbres M: A census of mitotic cancer genes: New insights into tumor cell biology and cancer therapy. Carcinogenesis 28: 899-912, 2007.

5. Tripathi A and Chaube SK: Reduction of phosphorylated Thr-161 Cdk1 level participates in roscovitine-induced Fas ligandmediated apoptosis in rat eggs cultured in vitro. In Vitro Cell Dev Biol Anim 51: 174-182, 2015.

6. Gray NS, Wodicka L, Thunnissen AM, Norman TC, Kwon S, Espinoza FH, Morgan DO, Barnes G, LeClerc S, Meijer L, et al: Exploiting chemical libraries, structure, and genomics in the search for kinase inhibitors. Science 281: 533-538, 1998.

7. Mori Y, Inoue Y, Taniyama Y, Tanaka S and Terada Y: Phosphorylation of the centrosomal protein, Cep169, by Cdk1 promotes its dissociation from centrosomes in mitosis. Biochem Biophys Res Commun 468: 642-646, 2015.

8. Iizuka D, Inanami O, Kashiwakura I and Kuwabara M: Purvalanol A enhances cell killing by inhibiting up-regulation of CDC2 kinase activity in tumor cells irradiated with high doses of $X$ rays. Radiat Res 167: 563-571, 2007.

9. Hsu HP, Li CF, Lee SW, Wu WR, Chen TJ, Chang KY, Liang SS Tsai CJ and Shiue YL: Overexpression of stathmin 1 confers an independent prognostic indicator in nasopharyngeal carcinoma. Tumour Biol 35: 2619-2629, 2014.

10. Baquero MT, Hanna JA, Neumeister V, Cheng H, Molinaro AM, Harris LN and Rimm DL: Stathmin expression and its relationship to microtubule-associated protein tau and outcome in breast cancer. Cancer 118: 4660-4669, 2012.

11. Akhtar J, Wang Z, Jiang WP, Bi MM and Zhang ZP: Stathmin overexpression identifies high risk for lymphatic metastatic recurrence in $\mathrm{pN} 0$ esophageal squamous cell carcinoma patients. J Gastroenterol Hepatol 29: 944-950, 2014.

12. Wegiel B, Wang Y, Li M, Jernigan F and Sun L: Novel indolylchalcones target stathmin to induce cancer cell death. Cell Cycle 15: 1288-1294, 2016.

13. Yip YY, Yeap YY, Bogoyevitch MA and Ng DC: Differences in c-Jun N-terminal kinase recognition and phosphorylation of closely related stathmin-family members. Biochem Biophys Res Commun 446: 248-254, 2014. 
14. Lu Y,Liu C, Xu YF, Cheng H, Shi S, Wu CT and Yu XJ: Stathmin destabilizing microtubule dynamics promotes malignant potential in cancer cells by epithelial-mesenchymal transition. Hepatobiliary Pancreat Dis Int 13: 386-394, 2014.

15. Akhtar J, Wang Z, Yu C, Li CS, Shi YL and Liu HJ: STMN-1 is a potential marker of lymph node metastasis in distal esophageal adenocarcinomas and silencing its expression can reverse malignant phenotype of tumor cells. BMC Cancer 14: 28, 2014.

16. Lin X, Liu S, Luo X, Ma X, Guo L, Li L, Li Z, Tao Y and Cao Y: EBV-encoded LMP1 regulates Op18/stathmin signaling pathway by $\mathrm{cdc} 2$ mediation in nasopharyngeal carcinoma cells. Int J Cancer 124: 1020-1027, 2009.

17. Lin X, Liao Y, Xie J, Liu S, Su L and Zou H: Op18/stathmin is involved in the resistance of Taxol among different epithelial carcinoma cell lines. Cancer Biother Radiopharm 29: 376-386, 2014.

18. Lin X, Liao Y, Chen X, Long D, Yu T and Shen F: Regulation of oncoprotein 18/stathmin signaling by ERK concerns the resistance to Taxol in nonsmall cell lung cancer cells. Cancer Biother Radiopharm 31: 37-43, 2016.

19. Laurie SA, Solomon BJ, Seymour L, Ellis PM, Goss GD, Shepherd FA, Boyer MJ, Arnold AM, Clingan P, Laberge F, et al: Randomised, double-blind trial of carboplatin and paclitaxel with daily oral cediranib or placebo in patients with advanced non-small cell lung cancer: NCIC Clinical Trials Group study BR29. Eur J Cancer 50: 706-712, 2014.

20. Wang T, Lv JH, Zhang XF, Li CJ, Han X and Sun YJ: Tissue inhibitor of metalloproteinase-1 protects MCF-7 breast cancer cells from paclitaxel-induced apoptosis by decreasing the stability of cyclin B1. Int J Cancer 126: 362-370, 2010.

21. Feng W, Xiaoyan X, Xuan Y, Xiangke L, Zichang Y, Ran Z, Liuxing W and Qingxia F: Silencing stathmin-modulating efficiency of chemotherapy for esophageal squamous cell cancer with paclitaxel. Cancer Gene Ther 22: 115-121, 2015.

22. Yared JA and Tkaczuk KH: Update on taxane development: New analogs and new formulations. Drug Des Devel Ther 6: 371-384, 2012.

23. Chen LK, Liang Y, Yang QY, Xu F, Zhou NN, Xu GC, Liu GZ and Wei WD: Triplet platinum-based combination sequential chemotherapy improves survival outcome and quality of life of advanced non-small cell lung cancer patients. Asian Pac J Cancer Prev 13: 1863-1867, 2012.

24. Lee HH, Ye S, Li XJ, Lee KB, Park MH and Kim SM: Combination treatment with paclitaxel and doxorubicin inhibits growth of human esophageal squamous cancer cells by inactivation of Akt. Oncol Rep 31: 183-188, 2014.
25. Kang J, Sergio CM, Sutherland RL and Musgrove EA: Targeting cyclin-dependent kinase 1 (CDK1) but not CDK4/6 or CDK2 is selectively lethal to MYC-dependent human breast cancer cells. BMC Cancer 14: 32, 2014.

26. Hayashi T, Adachi K, Ohba S and Hirose Y: The Cdk inhibitor flavopiridol enhances temozolomide-induced cytotoxicity in human glioma cells. J Neurooncol 115: 169-178, 2013.

27. Meng F, Bhupathi D, Sun JD, Liu Q, Ahluwalia D, Wang Y, Matteucci MD and Hart CP: Enhancement of hypoxia-activated prodrug TH-302 anti-tumor activity by Chk1 inhibition. BMC Cancer 15: 422, 2015.

28. Balasubramani M, Nakao C, Uechi GT, Cardamone J, Kamath K, Leslie KL, Balachandran R, Wilson L, Day BW and Jordan MA: Characterization and detection of cellular and proteomic alterations in stable stathmin-overexpressing, Taxol-resistant BT549 breast cancer cells using offgel IEF/PAGE difference gel electrophoresis. Mutat Res 722: 154-164, 2011.

29. Chen PW, Lin SJ, Tsai SC, Lin JH, Chen MR, Wang JT, Lee CP and Tsai $\mathrm{CH}$ : Regulation of microtubule dynamics through phosphorylation on stathmin by Epstein-Barr virus kinase BGLF4. J Biol Chem 285: 10053-10063, 2010.

30. Han ZX, Wang HM, Jiang G, Du XP, Gao XY and Pei DS: Overcoming paclitaxel resistance in lung cancer cells via dual inhibition of stathmin and Bcl-2. Cancer Biother Radiopharm 28: 398-405, 2013

31. Lin X, Tang M, Tao Y, Li L, Liu S, Guo L, Li Z, Ma X, Xu J and Cao Y: Epstein-Barr virus-encoded LMP1 triggers regulation of the ERK-mediated Op18/stathmin signaling pathway in association with cell cycle. Cancer Sci 103: 993-999, 2012.

32. He H, Sun YP, Zheng L and Yue ZG: Steroidal saponins from Paris polyphylla induce apoptotic cell death and autophagy in A549 human lung cancer cells. Asian Pac J Cancer Prev 16: $1169-1173,2015$

33. Wu Y, Tang M, Wu Y, Weng X, Yang L, Xu W, Yi W, Gao J, Bode AM, Dong Z, et al: A combination of paclitaxel and siRNA-mediated silencing of Stathmin inhibits growth and promotes apoptosis of nasopharyngeal carcinoma cells. Cell Oncol (Dordr) 37: 53-67, 2014.

34. Xi Q, Huang M, Wang Y, Zhong J, Liu R, Xu G, Jiang L, Wang J, Fang Z, Yang S: The expression of CDK1 is associated with proliferation and can be a prognostic factor in epithelial ovarian cancer. Tumour Biol 36: 4939-4948, 2015.

35. Yang JQ, Liu HX, Liang Z, Sun YM and Wu M: Over-expression of p53, p21 and Cdc2 in histologically negative surgical margins is correlated with local recurrence of laryngeal squamous cell carcinoma. Int J Clin Exp Pathol 7: 4295-4302, 2014. 